\title{
吡啶基离子液体 $\left[\mathrm{C}_{6} \mathrm{py}\right][\mathrm{DCA}]$ 热力学性质及利用新Eötvös方程预测其 表面张力
}

\author{
卜晓雪1 樊本汉 ${ }^{1}$ 魏 杰 ${ }^{1}$ 邢楠楠 ${ }^{1,2}$ 马晓雪 ${ }^{1}$ 关 伟 ${ }^{1, *}$ \\ ('辽宁大学化学院, 沈阳 110036; 2 黄山学院化学化工学院, 安徽 黄山 245041)
}

\begin{abstract}
摘要: 制备了吡啶类离子液体 $N$-已基吡啶二氰胺盐 $\left[\mathrm{C}_{6} \mathrm{Py}\right][\mathrm{DCA}]$, 并用核磁共振氢谱( $\left.{ }^{1} \mathrm{H} N \mathrm{NMR}\right)$ 、核磁共振碳 谱 $\left({ }^{13} \mathrm{C} N M R\right)$ 、差热扫描量热(DSC)、傅里叶变换红外 $(F T-I R)$ 光谱对其进行表征。在288.15-338.15 K温度范 围内, 采用标准加入法, 测定其密度 $(\rho)$ 、表面张力 $(y)$ 和折光率 $\left(n_{\mathrm{D}}\right)$ 。在测得的实验数据的基础上, 得到了离 子液体 $\left[\mathrm{C}_{6} \mathrm{py}\right][\mathrm{DCA}]$ 的分子体积 $\left(V_{\mathrm{m}}\right)$ 、表面能 $\left(E_{\mathrm{a}}\right)$ 、摩尔极化度 $\left(R_{\mathrm{m}}\right)$ 和极化率 $\left(\alpha_{\mathrm{p}}\right)$ 。结果显示 $E_{\mathrm{a}} 、 R_{\mathrm{m}}$ 和 $\alpha_{\mathrm{p}}$ 几乎不 随温度的变化而发生改变。本文还提出了摩尔表面Gibbs自由能 $\left(g_{\mathrm{s}}\right)$ 的概念, 并改进了Eötvös方程。同时还计 算了 $g_{\mathrm{s}}$ 、临界温度 $\left(T_{\mathrm{c}}\right)$ 和Eötvös方程经验参数 $\left(k_{\mathrm{E}}\right)$, 并预测了离子液体 $\left[\mathrm{C}_{6} \mathrm{py}\right][\mathrm{DCA}]$ 的表面张力, 预测值与实验 值具有较好的一致性。
\end{abstract}

关键词: 吡啶基离子液体; 密度; 表面张力; 摩尔表面Gibbs自由能; Eötvös方程 中图分类号: 0643

\section{Thermodynamic Properties and Predicting the Surface Tension of Pyridinium-Based lonic Liquids of [ $\mathrm{C}_{6}$ py][DCA] Using a New Eötvös Equation}

\author{
BU Xiao-Xue ${ }^{1} \quad$ FAN Ben-Han ${ }^{1} \quad$ WEI Jie ${ }^{1} \quad$ XING Nan-Nan $^{1,2} \quad$ MA Xiao-Xue $^{1} \quad$ GUAN Wei $^{1, *}$ \\ $\left({ }^{1}\right.$ College of Chemistry, Liaoning University, Shenyang 110036, P. R. China; ${ }^{2}$ College of Chemistry and Chemical Engineering, \\ Huangshang University, Huangshan 245041, Anhui Province, P. R. China)
}

\begin{abstract}
The pyridinium-based ionic liquids $\left[\mathrm{C}_{6} \mathrm{py}\right][\mathrm{DCA}](\mathrm{N}$-hexyl-pyridinium dicyanamide) was prepared and characterized using ${ }^{1} \mathrm{H}$ and ${ }^{13} \mathrm{C}$ nuclear magnetic responancec (NMR) spectroscopies, Fourier transform infrared (FT-IR) spectroscopy, and differential scanning calorimetry (DSC). The density ( $\rho$ ), surface tension $(\gamma)$, and refractive indices $\left(n_{\mathrm{D}}\right)$ were measured at the temperature range from 288.15 to $338.15 \mathrm{~K}$. Molecular volume $\left(V_{\mathrm{m}}\right)$, energy of surface $\left(E_{\mathrm{a}}\right)$, molar polarization $\left(R_{\mathrm{m}}\right)$, and polarization coefficient of [C $\mathrm{C}_{6}$ py] [DCA] $\left(\alpha_{\mathrm{p}}\right)$ were calculated from the experimental data. $E_{\mathrm{a}}, R_{\mathrm{m}}$, and $\alpha_{\mathrm{p}}$ were approximately temperature-independent. The concept of molar surface Gibbs free energy $\left(g_{\mathrm{s}}\right)$ was conceived, for which a new Eötvös equation was derived. The $g_{\mathrm{s}}$, critical temperature $\left(T_{\mathrm{c}}\right)$, and Eötvös empirical parameter related to polarity $\left(k_{\mathrm{E}}\right)$ were also obtained. The new Eötvös equation was used to predict the surface tension and the predicted values of $\left[\mathrm{C}_{6}\right.$ py] [DCA $]$ are in close agreement with the corresponding experimental ones.
\end{abstract}

Key Words: Pyridinium-based ionic liquid; Density; Surface tension; Molar surface Gibbs free energy; Eötvös equation

Received: September 9, 2015; Revised: October 28, 2015; Published on Web: October 30, 2015.

*Corresponding author. Email: guanweiy@sina.com.

The project was supported by the National Natural Science Foundation of China (21173107) and Liaoning Excellent Talents in University, China (2015025). 


\section{1 引 言}

近年来, 离子液体(ILs)因其独特的物理化学 性质如蒸气压小、热容大、导热性高、热稳定性 好、液态温度宽等而备受关注 ${ }^{1}$ 。这些性质使离子 液体在制药、物理和化学等诸多领域有着广阔的 应用前景 ${ }^{2-6}$ 。其中, 吡啶基离子液体除了具有咪唑 基离子液体的特性外, 还具有热稳定性好、热容 高、毒性低及价格更加低廉等特点 ${ }^{7-10}$, 这就使得其 在环境科学等研究领域得到了更多的关注 ${ }^{11-14}$ 。同 时, 含有二氧胺阴离子的离子液体可以用作新型 自燃材料和推进剂等 15,16 。

在工业生产中, 离子液体物理化学性质的基 础数据可以指导设备选型或工艺设计, 因而十分 重要。由于离子液体具有“可设计”性, 故合成并测 定所有离子液体的相关性质的基础数据是一项几 乎不可能实现的工程。如果能够预测所需离子液 体的物理化学性质, 并使其相关特性能够满足需 求, 就显得很重要了。近年来, 运用估算和预测 的方法研究物质的物理化学性质已成为一种发展 趋势, 尤其是在用半经验方法研究离子液体的相 关性质方面 ${ }^{17-20}$ 。

作为本课题组前期工作 ${ }^{21-25}$ 的延续, 本文报道 了如下工作：(1) 制备了 $N$-己基吡啶二氰胺盐离子 液体 $\left[\mathrm{C}_{6}\right.$ py $][\mathrm{DCA}]$; (2) 在288.15-338.15 K, 运用标 准加入法 $(\mathrm{SAM})^{21,25,26}$, 测定离子液体 $\left[\mathrm{C}_{6} \mathrm{py}\right][\mathrm{DCA}]$ 的 密度 $(\rho)$, 表面张力 $(\gamma)$ 和折光率 $\left(n_{\mathrm{D}}\right)$; (3) 计算了离 子液体 $\left[\mathrm{C}_{6} \mathrm{py}\right][\mathrm{DCA}]$ 的分子体积 $\left(V_{\mathrm{m}}\right)$ 、表面能 $\left(E_{\mathrm{a}}\right)$ 、 摩尔极化度 $\left(R_{\mathrm{m}}\right)$ 和极化率 $\left(\alpha_{\mathrm{p}}\right)$, 并讨论了这些性质 与温度的关系; (4) 提出了摩尔表面Gibbs自由能 $\left(g_{\mathrm{s}}\right)$ 的概念, 改进了Eötvös方程, 并预测了离子液 体 $\left[\mathrm{C}_{6} \mathrm{py}\right][\mathrm{DCA}]$ 的表面张力; (5) 计算了 $g_{s}$, 临界温 度 $\left(T_{\mathrm{c}}\right)$ 和Eötvös方程经验参数 $\left(k_{\mathrm{E}}\right)$ 。

\section{2 实验部分}

\section{1 实验药品}

吡定(> 99.9\%)、1-溴代已烷(> 99.9\%)、二氧胺 钠 $(>99 \%)$ 、硝酸银 $(>99 \%)$ 、乙酸乙酯 $(>99.9 \%)$ 均 购自于化学试剂国药控股有限公司。

\section{$2.2\left[\mathrm{C}_{6}\right.$ py][DCA] 的制备}

根据文献 ${ }^{25,27,28}$ 方法, 将等体积比的吡啶和乙酸 乙酯置于斜三口球瓶中, 再将适量的1-溴代已烷置 于恒压分液漏斗中。恒温至 $301.15 \mathrm{~K}$ 后, 向体系中 滴加1-溴代己烷, 反应 $72 \mathrm{~h}$ 。反应结束后, 加入乙
酸乙酯并搅拌, 重结晶, 直至生成白色颗粒, 真 空干燥 $48 \mathrm{~h}$ 。所得产物即为中间体 $\left[\mathrm{C}_{6} \mathrm{py}\right][\mathrm{Br}]$, 密 封保存。

称取一定量的 $\mathrm{AgNO}_{3}$ 和 $\mathrm{NaDCA}$ 溶解水中, 将 二氯胺钠溶液置于恒压分液漏斗中, 并滴加至硝 酸银溶液中。滴加完成后, 静置、分层、抽滤, 得到白色沉淀中间体 $\operatorname{AgDCA}$ 待用。将一定量的 $\left[\mathrm{C}_{6} \mathrm{py}\right][\mathrm{Br}]$ 溶于水中置于斜三口球瓶中, 加入上述 得到的白色中间体 $\operatorname{AgDCA}$, 搅拌 $24 \mathrm{~h}$ 。反应结束 后, 抽滤、旋蒸, 得到淡黄色离子液体 $\left[\mathrm{C}_{6} \mathrm{py}\right][\mathrm{DCA}]$, 干燥 $24 \mathrm{~h}$ 。

对合成产物进行 ${ }^{1} \mathrm{H} N \mathrm{NMR} 、{ }^{13} \mathrm{C}$ NMR、DSC、 FT-IR (见Supporting Information的图S1-S4及表 S1-S2)。经核磁共振谱分析没有发现杂质共振峰。 对 DSC 谱图分析, [ $\mathrm{C}_{6}$ py][DCA]的玻璃化转变温度 $T_{\mathrm{g}}$ 为 $-82.74{ }^{\circ} \mathrm{C}$ 。目标产物 $\left[\mathrm{C}_{6} \mathrm{py}\right][\mathrm{DCA}]$ 的纯度 $(\mathrm{HPCL} /$ 质量分数)为 $99.02 \%$ (见图 $\mathrm{S} 5$ )。

\section{$2.3\left[\mathrm{C}_{6}\right.$ py] $[\mathrm{DCA}]$ 密度、表面张力和折光率的测定}

考虑到 $N$ - 烷基 - 吡啶二氧胺离子液体 $\left[\mathrm{C}_{6} \mathrm{py}\right][\mathrm{DCA}]$ 与水能够形成很强的氢键 ${ }^{29}$, 使得用常 规的方法很难除尽其中的微量水, 这会对离子液 体 $\rho 、 \gamma$ 和 $n_{\mathrm{D}}$ 的测定产生影响。故采用标准加入法 (SAM) 来消除这种影响。用SFY-3000型微量水分 测定仪对 $\left[\mathrm{C}_{6} \mathrm{py}\right][\mathrm{DCA}]$ 的含水量 $w_{2}$ (质量分数)进行测 定, 根据 S A M , 配置一系列不同含水量的 $\left[\mathrm{C}_{6}\right.$ py $][\mathrm{DCA}]$ 样品。

本文使用安东帕DMA 4500密度计在288.15$338.15 \mathrm{~K}$ 下测定具有不同含水量的离子液体 $\left[\mathrm{C}_{6} \mathrm{py}\right][\mathrm{DCA}]$ 的密度, 测量精确度为 \pm 0.00005 $\mathrm{g} \cdot \mathrm{cm}^{-3}$, 控温精确度为 $\pm 0.03 \mathrm{~K}$ 。测定前对仪器进 行校正, 校正值与其文献值 ${ }^{30}$ 基本一致, 实验误差 为 $\pm 0.00001 \mathrm{~g} \cdot \mathrm{cm}^{-3}$ 。测定时, 样品在每个温度下测 定三次, 取平均值列于表 $\mathrm{S} 3$ 。将测定温度下的密 度值对含水量作图(见图S6), 能够得到拟合标准偏 差在实验误差范围内, 相关系数大于 0.99 的直线, 直线的截距为纯离子液体的密度, 相关数据均列 于表 $\mathrm{S} 3$ 中。

本文使用南京桑力 DP-AW表面张力实验装置 测定离子液体 $\left[\mathrm{C}_{6} \mathrm{py}\right][\mathrm{DCA}]$ 的表面张力, 测量精确 度为 $\pm 0.2 \mathrm{~mJ} \cdot \mathrm{m}^{-2}$ 。测定前, 对仪器进行校正, 校 正值与文献值 ${ }^{30}$ 基本一致。在288.15-338.15 K下, 分别测定一系列不同含水量的 $\left[\mathrm{C}_{6} \mathrm{py}\right][\mathrm{DCA}]$ 的表面 张力。升温时, 待温度达到预设值后恒温 $30 \mathrm{~min}$ 再 
开始读数。测定三次, 取平均值列于表 $\mathrm{S} 4$ 。将相 应温度下测得的表面张力对含水量作图(见图S7), 能得到很好的直线, 拟合系数大于 0.99 , 拟合标准 偏差在实验误差范围内, 截距为纯离子液体 [C 6 py $][\mathrm{DCA}]$ 的表面张力, 相关数据列于表 $\mathrm{S} 4$ 。

在288.15-338.15 K下，使用WYA-2W双目阿 贝折光仪测定离子液体 $\left[\mathrm{C}_{6} \mathrm{py}\right][\mathrm{DCA}]$ 的折光率, 测 量精确度为 \pm 0.0002 。实验前对仪器进行校正, 实 验误差为 \pm 0.0001 。测定时, 待温度升至预设值后 恒温 $30 \mathrm{~min}$ 再读数。测定三次, 取平均值, 列于表 $\mathrm{S} 5$ 中。将指定温度下测得的折光率对含水量作图 (见图S 8 ), 能得到拟合系数大于 0.99 的直线, 拟合 标准偏差在实验误差范围内, 截距为无水离子液 体 $\left[\mathrm{C}_{6} \mathrm{py}\right][\mathrm{DCA}]$ 的折光率, 相关数据列于表 $\mathrm{S} 5$ 中。

\section{3 结果与讨论}

\section{$3.1\left[\mathrm{C}_{6} \mathrm{py}\right][\mathrm{DCA}]$ 密度、表面张力和折光率}

根据 SAM，测定不同含水量 $\left[\mathrm{C}_{6} \mathrm{py}\right][\mathrm{DCA}]$ 的 $\rho 、 \gamma$ 和 $n_{\mathrm{D}}$, 通过线性回归外推得到纯离子液体, 即 不含水的离子液体的 $\rho$ 、 $\gamma$ 和 $n_{\mathrm{D}}$ 值。其值及其在 0.95 置信水平的实验扩展不确定度列于表 1 。

从表1中可以看出, 随着温度的升高, 离子液 体 $\left[\mathrm{C}_{6} \mathrm{py}\right][\mathrm{DCA}]$ 的 $\rho$ 、 $\gamma$ 及 $n_{\mathrm{D}}$ 值均减小。

\section{$3.2\left[\mathrm{C}_{6} \mathrm{py}\right][\mathrm{DCA}]$ 的体积性质}

根据上文测得的离子液体 $\left[\mathrm{C}_{6} \mathrm{py}\right][\mathrm{DCA}]$ 的 $\rho$, 可 以得到其分子体积 $V_{\mathrm{m}}$ (阴、阳离子体积之和 $)^{31}$, 计 算公式如下:

$$
V_{\mathrm{m}}=M /(N \cdot \rho)
$$

式中 $M$ 是离子液体的摩尔质量, $N$ 是 Avogadro常 数。根据公式(1)可计算出 $\left[\mathrm{C}_{6} \mathrm{py}\right][\mathrm{DCA}]$ 的 $V_{\mathrm{m}}$, 并将 计算结果列于表 2 中。为了讨论其与温度的关系, 以 $V_{\mathrm{m}}$ 为 $Y$ 轴, 温度 $(T)$ 为 $X$ 轴作图(见图1), 线性拟合

表1 在 288.15-338.15 K、0.1 MPa下, $\left[\mathrm{C}_{6} \mathrm{py}\right][\mathrm{DCA}]$ 的 $\rho$ 、 $\gamma$ 和 $n_{\mathrm{D}}$

Table 1 Density $(\rho)$, surface tension $(\gamma)$, and refractive index $\left(n_{\mathrm{D}}\right)$ for $\left[\mathrm{C}_{6} \mathrm{py}\right][\mathrm{DCA}]$ at the temperature range from 288.5 to 338.15 $\mathrm{K}$ and pressure of $0.1 \mathrm{MPa}$

\begin{tabular}{|c|c|c|c|}
\hline$T / \mathrm{K}$ & $\rho /\left(\mathrm{g} \cdot \mathrm{cm}^{-3}\right)$ & $\gamma /\left(\mathrm{mJ} \cdot \mathrm{m}^{-2}\right)$ & $n_{\mathrm{D}}$ \\
\hline 288.15 & $1.04237 \pm 0.00007$ & $49.9 \pm 0.3$ & $1.5298 \pm 0.0003$ \\
\hline 293.15 & $1.03938 \pm 0.00007$ & $49.6 \pm 0.3$ & $1.5282 \pm 0.0003$ \\
\hline 298.15 & $1.03641 \pm 0.00007^{*}$ & $49.2 \pm 0.3^{*}$ & $1.5267 \pm 0.0003^{*}$ \\
\hline 303.15 & $1.03341 \pm 0.00007$ & $48.9 \pm 0.3$ & $1.5253 \pm 0.0003$ \\
\hline 308.15 & $1.03043 \pm 0.00008$ & $48.5 \pm 0.3$ & $1.5238 \pm 0.0002$ \\
\hline 313.15 & $1.02746 \pm 0.00007$ & $48.2 \pm 0.3$ & $1.5221 \pm 0.0003$ \\
\hline 318.15 & $1.02452 \pm 0.00008$ & $47.9 \pm 0.3$ & $1.5205 \pm 0.0002$ \\
\hline 323.15 & $1.02158 \pm 0.00007$ & $47.7 \pm 0.3$ & $1.5191 \pm 0.0003$ \\
\hline 328.15 & $1.01865 \pm 0.00007$ & $47.4 \pm 0.3$ & $1.5176 \pm 0.0002$ \\
\hline 333.15 & $1.01575 \pm 0.00008$ & $47.1 \pm 0.3$ & $1.5160 \pm 0.0003$ \\
\hline 338.15 & $1.01286 \pm 0.00008$ & $46.8 \pm 0.3$ & $1.5144 \pm 0.0002$ \\
\hline
\end{tabular}

$u$ : standard uncertainty, $u(T)= \pm 0.01 \mathrm{~K}, u(p)= \pm 0.002 \mathrm{MPa} ;{ }^{*}$ Ref. 25

表2 288.15-338.15 K, [C 6 py][DCA $]$ 的 $V_{\mathrm{m}} 、 E_{\mathrm{a}} 、 R_{\mathrm{m}}$ 和 $\alpha_{\mathrm{p}}$

Table 2 Molecular volume $\left(V_{\mathrm{m}}\right)$, energy of surface $\left(E_{\mathrm{a}}\right)$, molar refraction $\left(R_{\mathrm{m}}\right)$, and polarization coefficient $\left(\alpha_{\mathrm{p}}\right)$ for $\left[\mathrm{C}_{6} \mathrm{py}\right][\mathrm{DCA}]$ from 288.15 to $338.15 \mathrm{~K}$

\begin{tabular}{ccccc}
\hline$T / \mathrm{K}$ & $V_{\mathrm{m}} / \mathrm{nm}^{3}$ & $E_{\mathrm{a}} /\left(\mathrm{mJ} \cdot \mathrm{m}^{-2}\right)$ & $R_{\mathrm{m}} /\left(\mathrm{cm}^{3} \cdot \mathrm{mol}^{-1}\right)$ & 68.24 \\
\hline 288.15 & 0.3670 & 67.7 & 68.26 & 27.07 \\
293.15 & 0.3680 & 67.7 & $68.30^{*}$ & 27.08 \\
298.15 & 0.3691 & 67.6 & 68.34 & 27.09 \\
303.15 & 0.3701 & 67.6 & 68.38 & 27.11 \\
308.15 & 0.3712 & 67.5 & 68.39 & 27.12 \\
313.15 & 0.3723 & 67.5 & 68.41 & 27.12 \\
318.15 & 0.3734 & 67.5 & 68.45 & 27.13 \\
323.15 & 0.3744 & 67.6 & 68.48 & 27.15 \\
328.15 & 0.3755 & 67.6 & 68.50 & 27.16 \\
333.15 & 0.3766 & 67.6 & 68.51 & 27.17 \\
338.15 & 0.3777 & 67.6 & 27.17 \\
\hline
\end{tabular}


得到一条斜率非常小的直线，这说明 $V_{\mathrm{m}}$ 随着 $T$ 的升 高而略微增大。

\section{$3.3\left[\mathrm{C}_{6}\right.$ py][DCA $]$ 的表面性质}

根据最小二乘法线性经验方程, 将利用SAM得 到纯离子液体 $\left[\mathrm{C}_{6} \mathrm{py}\right][\mathrm{DCA}]$ 的 $\gamma$ 值对 $T$ 作图(见图2), 拟合得到如下方程:

$$
\gamma=A_{0}-S_{\mathrm{a}} \cdot T
$$

式中 $A_{0}$ 是经验参数, $S_{\mathrm{a}}=-(\partial \gamma / \partial T)_{\mathrm{p}}$, 是表面熵。通过 计算得到 $\left[\mathrm{C}_{6} \mathrm{py}\right][\mathrm{DCA}]$ 的标准熵 $S_{\mathrm{a}}=61.6 \times 10^{-3}$ $\mathrm{mJ} \cdot \mathrm{K}^{-1} \cdot \mathrm{m}^{-2}$ 。

另外，在不同温度下的表面能 $E_{\mathrm{a}}$ 可通过纯离子 液体的表面张力值计算得到, 计算公式如下:

$$
E_{\mathrm{a}}=\gamma-T \cdot(\partial \gamma / \partial T)_{\mathrm{p}}
$$

将 $\left[\mathrm{C}_{6} \mathrm{py}\right][\mathrm{DCA}]$ 的表面能 $E_{\mathrm{a}}$ 值列于表 2 。为了讨

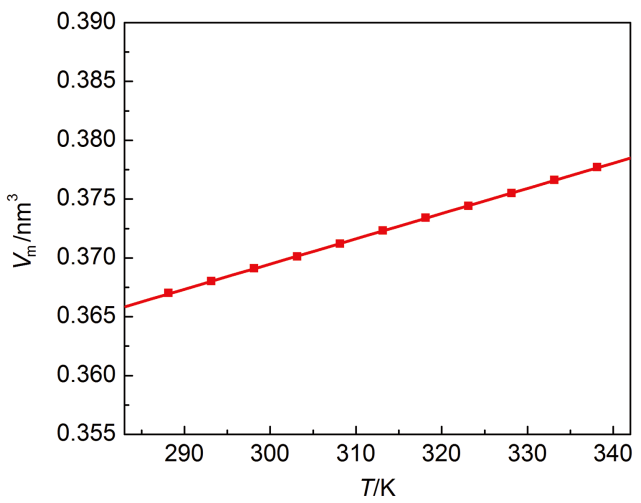

图1 288.15-338.15 K, [C $\left.\mathrm{C}_{6} \mathrm{py}\right][\mathrm{DCA}]$ 的 $V_{\mathrm{m}}$ 对 $T$ 作图

Fig.1 Plot of $V_{\mathrm{m}} v s T$ of ILs [C $\left.\mathrm{C}_{6} \mathrm{py}\right][\mathrm{DCA}]$ at the temperature range from 288.15 to $338.15 \mathrm{~K}$

$V_{\mathrm{m}}=0.30517+2.14364 \times 10^{-4} T, s=3.75 \times 10^{-5}, r=0.9999$ $s$ is the standard deviation of fitting.

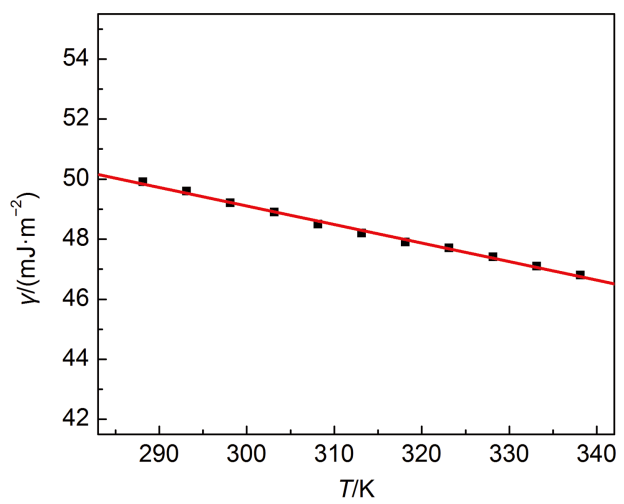

图2 288.15-338.15 K, [ $\left.\mathrm{C}_{6} \mathrm{py}\right][\mathrm{DCA}]$ 的 $\gamma$ 对 $T$ 作图

Fig.2 Plot of $\gamma$ vs $T$ of ILs [C 6 py] [DCA] at the temperature range from 288.15 to $338.15 \mathrm{~K}$

$\gamma=67.6-0.0616 T, s=0.07, r=0.998$
论表面能随温度的变化趋势, 将 $E_{\mathrm{a}}$ 对 $T$ 作图(见图 3 ), 拟合得到一条几乎平行于 $X$ 轴的直线。这表明 $E_{\mathrm{a}}$ 与温度无关, 而表面熵 $S_{\mathrm{a}}$ 是影响与温度有关的表 面性质的主要因素。

从表 2 的数据可以看出, 离子液体的表面能比 熔融盐要小，与有机液体更为接近 (如熔融 $\mathrm{NaNO}_{3}$ 的 $E_{\mathrm{a}}=146 \mathrm{~mJ} \cdot \mathrm{m}^{-2}$, 苯的 $E_{\mathrm{a}}=72.1 \mathrm{~mJ} \cdot \mathrm{m}^{-2}$, 正辛烷的 $\left.E_{\mathrm{a}}=51.1 \mathrm{~mJ} \cdot \mathrm{m}^{-2}\right)^{30,32}$ 。这说明, 与无机熔 融盐相比, 离子液体的离子间相互作用相对较 低, 因为 $E_{\mathrm{a}}$ 只取决于离子之间的相互作用能。

\section{$3.4\left[\mathrm{C}_{6} \mathrm{py}\right][\mathrm{DCA}]$ 的摩尔极化度和极化率}

由 $R_{\mathrm{m}}$ 和 $\alpha_{\mathrm{p}}$ 间的Lorentz-Lorenz关系，可得到 $R_{\mathrm{m}}$ 的定义式 ${ }^{33}$ :

$$
\begin{aligned}
R_{\mathrm{m}} & =\left[\left(n_{\mathrm{D}}^{2}-1\right) /\left(n_{\mathrm{D}}^{2}+2\right)\right] \cdot(M / \rho) \\
& =(4 \pi N / 3) \cdot \alpha_{\mathrm{p}}
\end{aligned}
$$

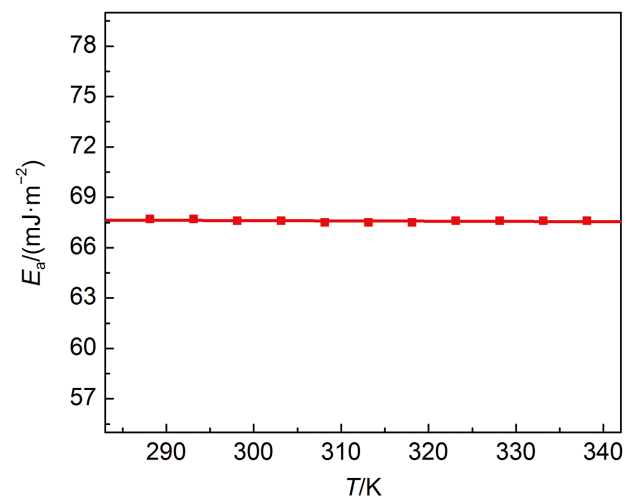

图3 288.15-338.15 K, [C 6 py][DCA] 的 $E_{\mathrm{a}}$ 对 $T$ 作图

Fig.3 Plot of $E_{\text {a }} v s T$ of ILs [C 6 py][DCA] at the temperature range from 288.15 to $338.15 \mathrm{~K}$

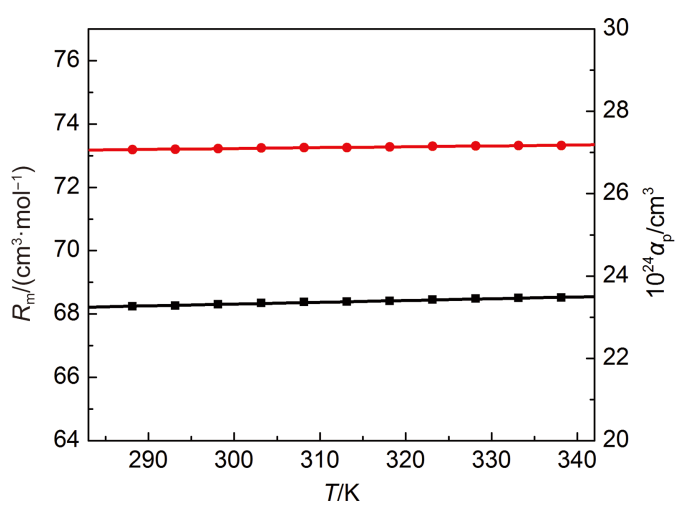

图4 288.15-338.15 K, $\left[\mathrm{C}_{6} \mathrm{py}\right][\mathrm{DCA}]$ 的 $R_{\mathrm{m}}$ 和 $10^{24} \alpha_{\mathrm{p}}$ 分别对 $T$ 作图

Fig.4 Plots of $R_{\mathrm{m}}$ and the polarization coefficient $\left(10^{24} \alpha_{\mathrm{p}}\right)$ vs $T$ of ILs $\left[\mathrm{C}_{6} \mathrm{py}\right][\mathrm{DCA}]$ at the temperature range from 288.15 to $338.15 \mathrm{~K}$

- $R_{\mathrm{m}}=66.6+0.00564 T ; \bullet 10^{24} \alpha_{\mathrm{p}}=26.46+0.00211 T$ 
根据上式，可以计算出在288.15-338.15 K温度范 围内，离子液体 $\left[\mathrm{C}_{6} \mathrm{py}\right][\mathrm{DCA}]$ 的 $R_{\mathrm{m}}$ 和 $\alpha_{\mathrm{p}}$, 并将结果 列于表 2 中。分别将得到的 $R_{\mathrm{m}}$ 和 $\alpha_{\mathrm{p}}$ 值对 $T$ 做图(见图 $4)$, 拟合后可以看出, $R_{\mathrm{m}}$ 和 $\alpha_{\mathrm{p}}$ 的值与温度无关, $R_{\mathrm{m}}$ 和 $\alpha_{\mathrm{p}}$ 反映了离子液体的诱导偶极作用。

\section{5 用Eötvös方程预测离子液体 $\left[\mathrm{C}_{6} \mathrm{py}\right][\mathrm{DCA}]$ 的} 表面张力

对于大多数的离子液体, 其表面张力值随着 温度的升高而减小，其关系可用下列Eötvös方程 ${ }^{32,34}$ 表示:

$$
\gamma V^{2 / 3}=k_{\mathrm{E}}\left(T_{\mathrm{c}}-T\right)
$$

式中 $V$ 是摩尔体积, $\gamma$ 是表面张力, $k_{\mathrm{E}}$ 是Eötvös 经验 参数, $k_{\mathrm{E}}$ 与极性有关。将离子液体 $\left[\mathrm{C}_{6} \mathrm{py}\right][\mathrm{DCA}]$ 的 $\gamma V^{2 / 3}$ 对 $T$ 作图(见图5), 拟合得到一条直线。直线的 斜率即 $k_{\mathrm{E}}$ 。其中, $\left[\mathrm{C}_{6} \mathrm{py}\right][\mathrm{DCA}]$ 的 $k_{\mathrm{E}}$ 值为 $1.6 \times 10^{-7}$ $\mathrm{J} \cdot \mathrm{mol}^{-2 / 3} \cdot \mathrm{K}^{-1}$ 。有机溶剂的极性低, $k_{\mathrm{E}} \approx 2.2 \times 10^{-7}$ $\mathrm{J} \cdot \mathrm{mol}^{-2 / 3} \cdot \mathrm{K}^{-1}, \mathrm{NaCl}$ 熔融盐极性高, $k_{\mathrm{E}} \approx 0.4 \times 10^{-7}$ $\mathrm{J} \cdot \mathrm{mol}^{-2 / 3} \cdot \mathrm{K}^{-1}$ 。由此可以看出, 离子液体的极性低 于 $\mathrm{NaCl}$ 熔融盐, 但高于有机溶剂。另外, 随着碳 链的增长, $k_{\mathrm{E}}$ 值微增, 也就是说离子液体的极性随 着碳链的增长而降低。

Eötvös经验方程可以很好地解释表面张力和温 度的关系, 但是其中 $\gamma V^{2 / 3}$ 的物理意义不明确且其单

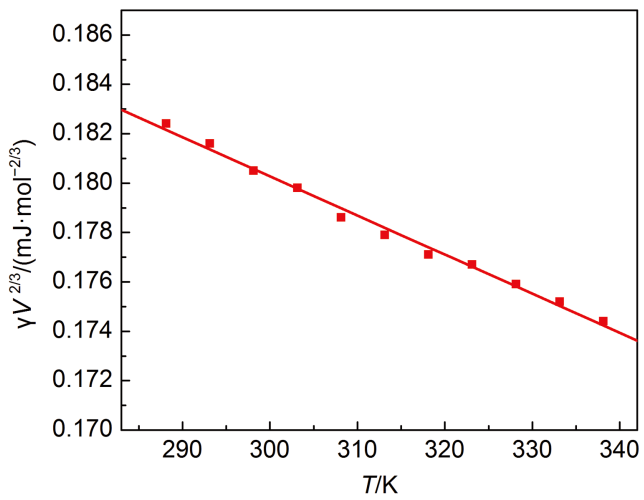

图5 288.15-338.15 K, [C 6 py][DCA]的 $\gamma V^{2 / 3}$ 对 $T$ 作图

Fig.5 Plot of $\gamma V^{2 / 3}$ vs $T$ of [C $C_{6}$ py][DCA] at the temperature range from 288.15 to $338.15 \mathrm{~K}$

$\gamma V^{2 / 3}=0.2280-1.59 \times 10^{-4} T, s=2.35 \times 10^{-4}, r=0.996$
位 $\mathrm{J} \cdot \mathrm{mol}^{-2 / 3}$ 也不方便使用。故本文提出了摩尔表面 Gibbs 自由能概念并对Eötvös经验方程进行了改 进。

摩尔表面Gibbs自由能 $g_{\mathrm{s}}$ 可定义为

$g_{\mathrm{s}}=\gamma V^{2 / 3} N^{1 / 3}$

将公式(6)代入公式(5), 摩尔表面Gibbs自由能 和温度的关系可用下列经验方程表示:

$$
\begin{aligned}
g_{\mathrm{s}} & =k_{\mathrm{E}} N^{1 / 3}\left(T_{\mathrm{c}}-T\right) \\
& =k_{\mathrm{E}} N^{1 / 3} T_{\mathrm{c}}-k_{\mathrm{E}} N^{1 / 3} T=C_{0}-C_{1} T
\end{aligned}
$$

式中 $C_{i}$ 是经验参数, $C_{1}=-k_{\mathrm{E}} N^{1 / 3}, C_{0}=k_{\mathrm{E}} N^{1 / 3} T_{\mathrm{c}}, T_{\mathrm{c}}=$ $-C_{0} / C_{1}$ 。由方程(6)计算得到的离子液体 $\left[\mathrm{C}_{6} \mathrm{py}\right][\mathrm{DCA}]$ 的 $g_{\mathrm{s}}(\exp )$ 值对 $T$ 作图, 得到一条很好的直线(见图 $6)$, 直线的拟合系数均大于 0.99 , 标准偏差值在实 验误差范围内。参数 $C_{i}$ 值, 标准偏差 $s$, 相关系数 $r$ 及通过计算得到的离子液体的临界温度 $T_{\mathrm{c}}$ 值均列 于表 $3 ， g_{s}(\exp )$ 值列于表4。

当温度升至临界温度 $T_{\mathrm{c}}$ 时, 摩尔表面Gibbs自 由能减至 0 , 这样就从方程(7)变形得到了新的 Eötvös方程:

$$
g_{\mathrm{s}}=C_{1}\left(T_{\mathrm{c}}-T\right)
$$

上式为改进的Eötvös方程, 参数 $C_{1}$ 和 $k_{\mathrm{E}}$ 的关系 与传统方程一样, $C_{1}=N^{1 / 3} k_{\mathrm{E}}$, 单位是 $\mathrm{J} \cdot \mathrm{mol}^{-1} \cdot \mathrm{K}^{-1}$ 。 尽管方程(5)和方程(8)都是经验方程, 但后者中的

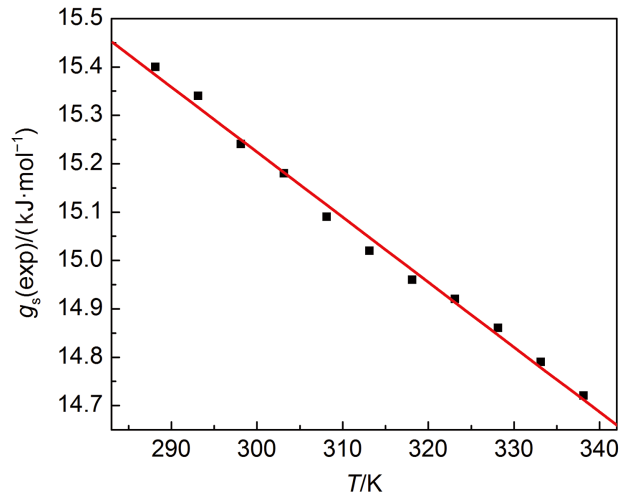

图6 288.15-338.15 K, [ $\left.\mathrm{C}_{6} \mathrm{py}\right][\mathrm{DCA}]$ 的 $\mathrm{g}_{\mathrm{s}}$ 对 $T$ 作图

Fig.6 Plot of $g_{s} v s T$ of $\left[\mathrm{C}_{6} \mathrm{py}\right][\mathrm{DCA}]$ at the temperature range from 288.15 to $338.15 \mathrm{~K}$

$g_{\mathrm{s}}=19.25-0.01344 T, s=0.02, r=0.997, g_{\mathrm{s}}(\exp )$ is experimental values of the molar surface Gibbs free energy.

表3 从方程(7)得到的 $C_{0}, C_{1}, r, s$ 和 $T_{\mathrm{c}}$ 参数值

Table 3 Parameter values of $C_{0}, C_{1}, r, s$, and $T_{\mathrm{c}}$ by empirical Eq.(7)

\begin{tabular}{ccccc}
\hline $\mathrm{IL}$ & $C_{0} /\left(\mathrm{J} \cdot \mathrm{mol}^{-1}\right)$ & $C_{1} /\left(\mathrm{J} \cdot \mathrm{mol}^{-1} \cdot \mathrm{K}^{-1}\right)$ & $r$ & $s /\left(\mathrm{J} \cdot \mathrm{mol}^{-1}\right)$ \\
\hline$\left[\mathrm{C}_{6}\right.$ py $][\mathrm{DCA}]$ & 19.25 & 0.013 & 0.997 & 0.013 \\
\hline$C_{0}, C_{1}$ are parameters; $r$ is the correlation coefficient of fitting; $T_{\mathrm{c}}$ is critical temperature.
\end{tabular}

$C_{0}, C_{1}$ are parameters; $r$ is the correlation coefficient of fitting; $T_{\mathrm{c}}$ is critical temperature. 
每一项均有明显的物理意义和能量单位, 这样更 便于应用。在这个改进中, 可以注意到方程将摩 尔表面Gibbs自由能与液体的体积、密度和表面张 力联系在一起, 因此它可以发展为一种用来预测 物质的物理性质的方法。

将方程(8)中的 $C_{1}$ 和 $T_{\mathrm{c}}$ 代入后，可以用来估算指 定温度下离子液体的摩尔表面Gibbs自由能 $g_{\mathrm{s}}$ (est), $g_{\mathrm{s}}(\mathrm{est})$ 的估算值列于表4。再根据方程(6), 就可以 用得到的 $g_{\mathrm{s}}(\mathrm{est})$ 值来估算离子液体的表面张力, $\gamma($ est)的估算值列于表 4 。将表面张力估算值 $\gamma($ est $)$ 对 其实验值 $\gamma(\exp )$ 作图, 就可得到一条较好的直线(见 图7)。拟合直线的斜率接近于 1 , 拟合相关系数 $r=$ 0.999 。上述事实说明表 4 中的 $\gamma($ est $)$ 与表 1 中的 $\gamma(\exp )$ 能够较好的吻合。

\section{表4 288.15-338.15 K, [C 6 py][DCA $]$ 的 $g_{s}(\exp ) 、 g_{s}(\mathrm{est})$ 及 $\gamma($ est) 值}

Table $4 g_{s}(\exp ), g_{s}($ est $)$, and $\gamma\left(\right.$ est) for $\left[C_{6}\right.$ py] [DCA] at the temperature range from 288.15 to $338.15 \mathrm{~K}$

\begin{tabular}{cccc}
\hline$T / \mathrm{K}$ & $g_{\mathrm{s}}(\mathrm{exp}) /\left(\mathrm{kJ} \cdot \mathrm{mol}^{-1}\right)$ & $g_{\mathrm{s}}(\mathrm{est}) /\left(\mathrm{kJ} \cdot \mathrm{mol}^{-1}\right)$ & $\gamma(\mathrm{est}) /\left(\mathrm{mJ} \cdot \mathrm{m}^{-2}\right)$ \\
\hline 288.15 & 15.40 & 15.37 & 49.8 \\
293.15 & 15.34 & 15.30 & 49.5 \\
298.15 & 15.24 & 15.23 & 49.2 \\
303.15 & 15.18 & 15.17 & 48.9 \\
308.15 & 15.09 & 15.10 & 48.5 \\
313.15 & 15.02 & 15.03 & 48.2 \\
318.15 & 14.96 & 14.96 & 47.9 \\
323.15 & 14.92 & 14.90 & 47.6 \\
328.15 & 14.86 & 14.83 & 47.3 \\
333.15 & 14.79 & 14.76 & 47.0 \\
338.15 & 14.72 & 14.70 & 46.7 \\
\hline
\end{tabular}

$g_{\mathrm{s}}(\mathrm{est})$ is the predicted values of the molar surface Gibbs free energy, $\gamma(\mathrm{est})$ is the predicted values of surface tension.

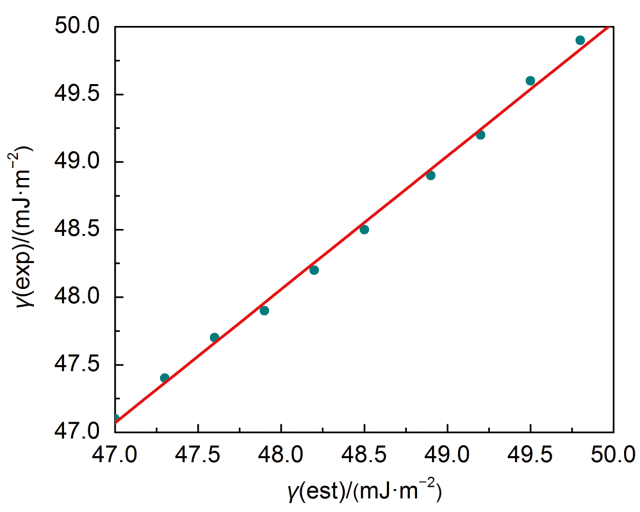

图7 表面张力的实验值 $\gamma(\exp )$ 对估算值 $\gamma($ est)作图

Fig.7 Plot of experimental $\gamma(\exp )$ values vs estimated $\gamma($ est) values

$\gamma(\exp )=0.7+0.98 \gamma(\mathrm{est}), s=0.05, r=0.999$

\section{4 结 论}

计算得到了离子液体 $\left[\mathrm{C}_{6} \mathrm{py}\right][\mathrm{DCA}]$ 的 $V_{\mathrm{m}} 、 E_{\mathrm{a}}$ 、 $R_{\mathrm{m}} 、 \alpha_{\mathrm{p}}$, 其中, $V_{\mathrm{m}}$ 随着温度的升高略微增大, $E_{\mathrm{a}}$ 、 $R_{\mathrm{m}}$ 和 $\alpha_{\mathrm{p}}$ 并不随温度的变化发生改变; 本文还提出了 摩尔表面积Gibbs自由能 $g_{s}$ 的概念, 并对Eötvös方程 进行改进, 改进后的Eötvös方程的每一项物理量具 有明确的物理意义，并可以与液体体积、密度、 表面张力等联系一起, 可以用来预测物质的物理 化学性质。用新型Eötvös方程预测的离子液体 $\left[\mathrm{C}_{6} \mathrm{py}\right][\mathrm{DCA}]$ 的表面张力值与实验值具有较好的一 致性。

Supporting Information: available free of charge via the internet at http://www.whxb.pku.edu.cn.

\section{References}

(1) Seddon, K. R. J. Chem. Tech. Biotechnol. 1997, 68 (4), 351.

(2) Tao, G. H.; He, L.; Liu, W. S.; Xu, L.; Xiong, W.; Wang, T.; Kou, Y. Green Chem. 2006, 8, 639. doi: 10.1039/b600813e

(3) Muhammad, N.; Omar, W. N.; Man, Z.; Bustam, M. A.; Rafiq, S.; Uemura, Y. Ind. Eng. Chem. Res. 2012, 51 (5), 2280. doi: 10.1021/ie2014313

(4) Rout, A.; Binnemans, K. Ind. Eng. Chem. Res. 2014, 53 (8), 6500.

(5) Zhong, H. X.; Zhao, C. B.; Luo, H.; Zhang, L. Z. Acta Phys. -Chim. Sin. 2012, 28 (11), 2641. [仲皓想, 赵春宝, 骆 浩, 张灵志. 物理化学学报, 2012, 28 (11), 2641.] doi: 10.3866/PKU.WHXB201207181

(6) Wang, H.; Xu, X. Q.; Shi, J. F.; Xu, G. Acta Phys. -Chim. Sin. 2013, 29 (3), 525. [王 海, 徐雪青, 史继富, 徐 刚. 物理化学 学报, 2013, 29 (3), 525.] doi: 10.3866/PKU.WHXB201301091

(7) Zhao, D. B.; Fei, Z. F.; Geldbach, T. J.; Scopelliti, R.; Dyson, P. J. J. Am. Chem. Soc. 2004, 126 (48), 15876. doi: 10.1021/ja0463482

(8) Yunus, N. M.; Abdul Mutalib, M. I.; Man, Z.; Bustam, M. A.; Murugesan, T. Chemical Engineering Journal 2012, 189, 94.

(9) Calvar, N.; Gomez, E.; Macedo, E. A.; Dominguez, A. Thermochimica Acta 2013, 565, 178. doi: 10.1016/j.tca.2013.05.007

(10) Crosthwaite, J. M.; Muldoon, M. J.; Dixon, J. K.; Anderson, J. L.; Brennecke, J. F. Journal of Chemical Thermodynamics 2005, 37 (6), 559. doi: 10.1016/j.jct.2005.03.013

(11) Xu, F.; Gao, H. S.; Dong, H. F.; Wang, Z. L.; Zhang, X. P.; Ren, B. Z.; Zhang, S. J. Fluid Phase Equilibria 2014, 365, 80. doi: 10.1016/j.fluid.2013.12.020

(12) Ye, Q.; Gao, T. T.; Wan, F.; Yu, B.; Pei, X. M.; Zhou, F.; Xue, Q. J. Journal of Materials Chemistry 2012, 22 (26), 13123. doi: 10.1039/c2jm31527k

(13) Zeng, S. J.; Gao, H. S.; Zhang, X. C.; Dong, H. F.; Zhang, X. P.; 
Zhang, S. J. Chemical Engineering Journal 2014, 251, 248. doi: 10.1016/j.cej.2014.04.040

(14) Jie, X. M.; Chau, J.; Obuskovic, G.; Obuskovic, G.; Sirkar, K. K. Industrial \& Engineering Chemistry Research 2014, 53 (8), 3305. doi: 10.1021/ie403596b

(15) Schneider, S.; Hawkins, T.; Rosander, M.; Vaghjiani, G.; Chambreau, S.; Drake, G. Energy \& Fuels 2008, 22 (4), 2871. doi: 10.1021/ef800286b

(16) Bedrov, D.; Borodin, O. Journal of Physical Chemistry B 2010, 114 (40), 12802. doi: 10.1021/jp1049827

(17) Tokuda, H.; Hayamizu, K.; Ishii, K.; Susan, M. A. B. H.; Watanabe, M. Journal of Physical Chemistry B 2004, 108 (42), 16593. doi: 10.1021/jp047480r

(18) Krossing, I.; Slattery, J. M.; Daguenet, C.; Dyson, P. J.; Oleinikova, A.; Weingärtner, H. J. Am. Chem. Soc. 2006, 128 (41), 13427

(19) Jenkins, H. D. B.; Glasser, L. Inorganic Chemistry 2002, 41 (17), 4378. doi: $10.1021 /$ ic020222t

(20) Jenkins, H. D. B.; Glasser, L. Inorganic Chemistry 2003, 42 (26), 8702. doi: $10.1021 / \mathrm{ic} 030219 \mathrm{p}$

(21) Ma, X. X.; Wei, J.; Zhang, Q. B.; Tian, F.; Feng, Y. Y.; Guan, W. Ind. Eng. Chem. Res. 2013, 52, 9490. doi: 10.1021/ie401130d

(22) Wei, J.; Chang, C.; Zhang, Y. Y.; Hou, S. Y.; Fang, D. W.; Guan, W. J. Chem. Thermodynamics 2015, 90, 310.

(23) Wei, J.; Zhang, Q. B.; Tian, F.; Zheng, L.; Guan, W.; Yang, J. Z. Fluid Phase Equilibria 2014, 371, 1. doi: 10.1016/j.fluid.2014.03.011

(24) Guan, W.; Zhang, Q. B.; Ma, X. X.; Wei, J.; Pan, Y.; Yang, J. Z.
Fluid Phase Equilibria 2013, 360, 63. doi: 10.1016/j.fluid.2013.09.032

(25) Ma, X. X.; Wei, J.; Guan, W.; Pan, Y.; Zheng, L.; Wu, Y.; Yang, J. Z. J. Chem. Thermodynamics 2015, 89, 51. doi: 10.1016/j.jct.2015.02.025

(26) Guan, W.; Wang, C. X.; Wang, Z.; Chen, S. P.; Gao, S. L. Acta Chim. Sin. 2011, 69 (11), 1280. [关＼cjkstart伟, 王彩霞, 王 珍, 陈三 平, 高胜利. 化学学报, 2011, 69 (11), 1280.]

(27) Earle, M. J.; Gordon, C. M.; Plechkova, N. V.; Seddon, K. R.; Welton, T. Analytical Chemistry 2007, 79 (2), 758. doi: 10.1021/ac061481t

(28) Gordon, C. M.; Muldoon, M. J.; Wagner, M. Ionic Liquids in Synthesis; Wiley-VCH Verlag GmbH \& Co. KGaA: Weinheim, 2002.

(29) Zhang, S. G.; Qi, X. J.; Ma, X. Y.; Lu, L. J.; Deng, Y. Q. J. Phys. Chem. B 2010, 114 (11), 3912. doi: 10.1021/jp911430t

(30) Lide, D. R. Handbook of Chemistry and Physics, 82nd ed.; CRC Press: Boca Raton, FL, 2001.

(31) Glasser, L. Thermochimica Acta 2004, 421 (1-2), 87. doi: 10.1016/j.tca.2004.03.015

(32) Adamson, A. W. Physical Chemistry of Surfaces, 3rd ed.; John Wiley: New York, 1976; translated by Gu, T. R. Science Press: Beijing, 1986. [Adamson, A. W. 表面物理化学. 第三版. 顾惕人 译. 北京: 科学出版社, 1986.]

(33) Ersfeld, B.; Felderhof, B. U. Phys. Rev. E 1998, 57 (1), 1118. doi: 10.1103/PhysRevE.57.1118

(34) Tong, J.; Liu, Q. S.; Zhang, P.; Yang, J. Z. J. Chem. Eng. Data 2007, 52 (4), 1497. doi: 10.1021/je700102g 
Supplementary Information for Acta Phys. -Chim. Sin. 2016, 32 (1), 267-273

doi: $\quad$ 10.3866/PKU.WHXB201510303

\title{
吡啶基离子液体 $\left[\mathrm{C}_{6} \mathrm{py}\right][\mathrm{DCA}]$ 热力学性质及利用新 Eötvös 方 程预测其表面张力
}

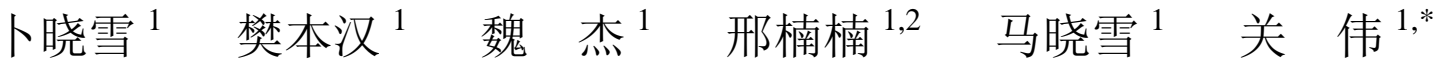
('辽宁大学化学院, 沈阳 110036; 2 黄山学院化学化工学院, 安徽 黄山 245041)

\section{Thermodynamic Properties and Predicting the Surface Tension of Pyridinium-Based Ionic Liquids of [ $\left.\mathrm{C}_{6} \mathrm{py}\right][\mathrm{DCA}]$ Using a New Eötvös Equation}

\author{
BU Xiao-Xue $^{1} \quad$ FAN Ben-Han $^{1} \quad$ WEI Jie $^{1} \quad$ XING Nan-Nan $^{1,2} \quad$ MA Xiao-Xue $^{1}$ \\ GUAN Wei ${ }^{1, *}$ \\ $\left({ }^{1}\right.$ College of Chemistry, Liaoning University, Shenyang 110036, P. R. China; $\quad{ }^{2}$ College of Chemistry and \\ Chemical Engineering, Huangshang University, Huangshan 245041, Anhui Province, P. R. China)
}

*Corresponding author. Email: guanweiy@sina.com. 
一、表征

A $\quad\left[\mathrm{C}_{6} \mathrm{py}\right][\mathrm{DCA}]$ 的 ${ }^{1} \mathrm{H}$ NMR 表征

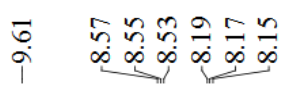

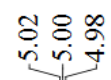

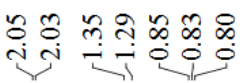

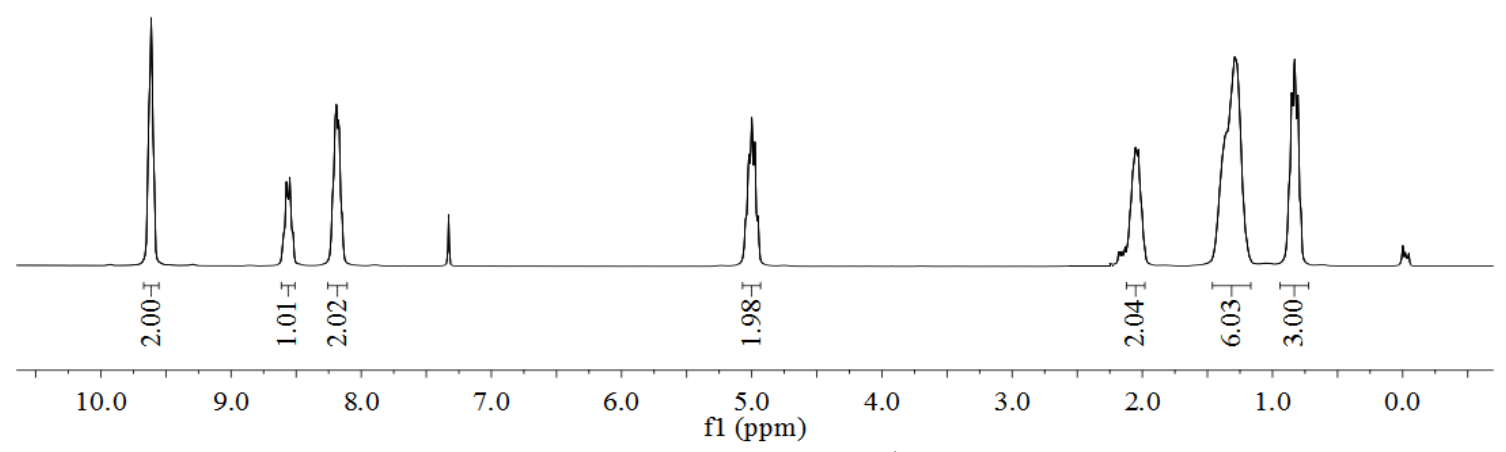

图 S1 离子液体 $\left[\mathrm{C}_{6} \mathrm{py}\right][\mathrm{DCA}]$ 的 ${ }^{1} \mathrm{H} \mathrm{NMR}$ 谱图

Fig.S1 ${ }^{1} \mathrm{H}$ NMR of IL [C 6 py][DCA]

表 S1 [C 6 py][DCA] 的 ${ }^{1} \mathrm{H}$ NMR 的化学位移 (300 $\mathrm{MHz}, \mathrm{CDCl}_{3}$ )

Table S1 The ${ }^{1} \mathrm{H}$ NMR spectrum $\delta_{\mathrm{H}}\left(300 \mathrm{MHz}, \mathrm{CDCl}_{3}\right)$ of $\left[\mathrm{C}_{6} \mathrm{py}\right][\mathrm{DCA}]$

\begin{tabular}{ccc} 
Chemical shift (ppm) & Hydrogen number & Radical \\
\hline $0.78(\mathrm{t})$ & 3 & $\mathrm{CH}_{2} \mathrm{CH}_{2} \boldsymbol{C H}_{3}$ \\
$1.28-1.22(\mathrm{~m})$ & 6 & $\mathrm{CH}_{2} \boldsymbol{C H}_{2} \boldsymbol{C H}_{2} \boldsymbol{C H}_{2} \mathrm{CH}_{3}$ \\
$1.98-1.96(\mathrm{~m})$ & 2 & $\mathrm{NCH}_{2} \boldsymbol{C H}_{2} \mathrm{CH}_{2}$ \\
$4.95(\mathrm{t})$ & 2 & $\mathrm{NCH}_{2}$ \\
$8.12(\mathrm{t})$ & 2 & $\mathrm{C}(2) \boldsymbol{H} 、 \mathrm{C}(4) \boldsymbol{H}$ \\
$8.51(\mathrm{t})$ & 1 & $\mathrm{C}(3) \boldsymbol{H}$ \\
$9.55(\mathrm{~d})$ & 2 & $\mathrm{C}(1) \boldsymbol{H} 、 \mathrm{C}(5) \boldsymbol{H}$ \\
\hline
\end{tabular}

注: d: 二重峰; $\mathrm{t}$ : 三重峰; $\mathrm{m}$ : 多重峰。 


\section{B $\quad\left[\mathrm{C}_{6} \mathrm{py}\right][\mathrm{DCA}]$ 的 ${ }^{13} \mathrm{C}$ NMR 表征}

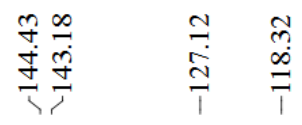

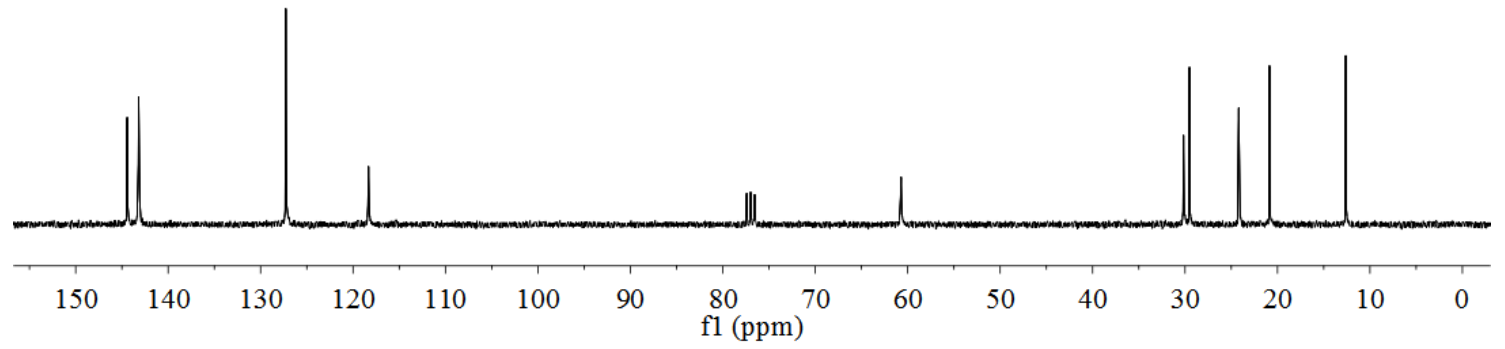

图 S2 离子液体 $\left[\mathrm{C}_{6} \mathrm{py}\right][\mathrm{DCA}]$ 的 ${ }^{13} \mathrm{C} \mathrm{NMR}$ 谱图 Fig.S2 ${ }^{13} \mathrm{C}$ NMR of IL [C 6 py][DCA]

表 S2 [C 6 py][DCA]的 ${ }^{13} \mathrm{C} \mathrm{NMR}$ 的化学位移（70 $\mathrm{MHz}, \mathrm{CDCl}_{3}$ )

Table S2 The ${ }^{13} \mathrm{C}$ NMR spectrum $\delta_{\mathrm{C}}\left(70 \mathrm{MHz}, \mathrm{CDCl}_{3}\right)$ of $\left[\mathrm{C}_{6} \mathrm{py}\right][\mathrm{DCA}]$

\begin{tabular}{cc} 
Chemical shift (ppm) & Radical \\
\hline 12.61 & $-\mathrm{CH}_{3}$ \\
$20.87 \sim 30.16$ & $-\mathrm{CH}_{2}-$ \\
60.80 & $-\mathrm{CH}_{2}-\mathrm{N}-$ \\
118.32 & $=\mathrm{CH}-$ \\
127.12 & $=\mathrm{CH}-$ \\
143.18 & $=\mathrm{CH}-\mathrm{N}-$ \\
144.43 & $-\mathrm{C} \equiv \mathrm{N}$
\end{tabular}


C 傅里叶变换红外光谱 (FT-IR) 表征

红外光谱谱图数据的大致归属: $\mathrm{C}=\mathrm{C}$ 的骨架振动 $v_{\mathrm{C}=\mathrm{C}}$ 位于 $1500-1600 \mathrm{~cm}^{-1}, \mathrm{C}-\mathrm{N}$ 的伸缩振 动 $v_{=\mathrm{C}-\mathrm{N}}$ 位于 $1700-1900 \mathrm{~cm}^{-1}, \mathrm{C}-\mathrm{H}$ 的伸缩振动 $v_{=\mathrm{C}-\mathrm{H}}$ 位于 $2900-3200 \mathrm{~cm}^{-1},-\mathrm{C} \equiv \mathrm{N}$ 的伸缩振动 $v_{\mathrm{C} \equiv \mathrm{N}}$ 位 于2050-2250 $\mathrm{cm}^{-1}$.

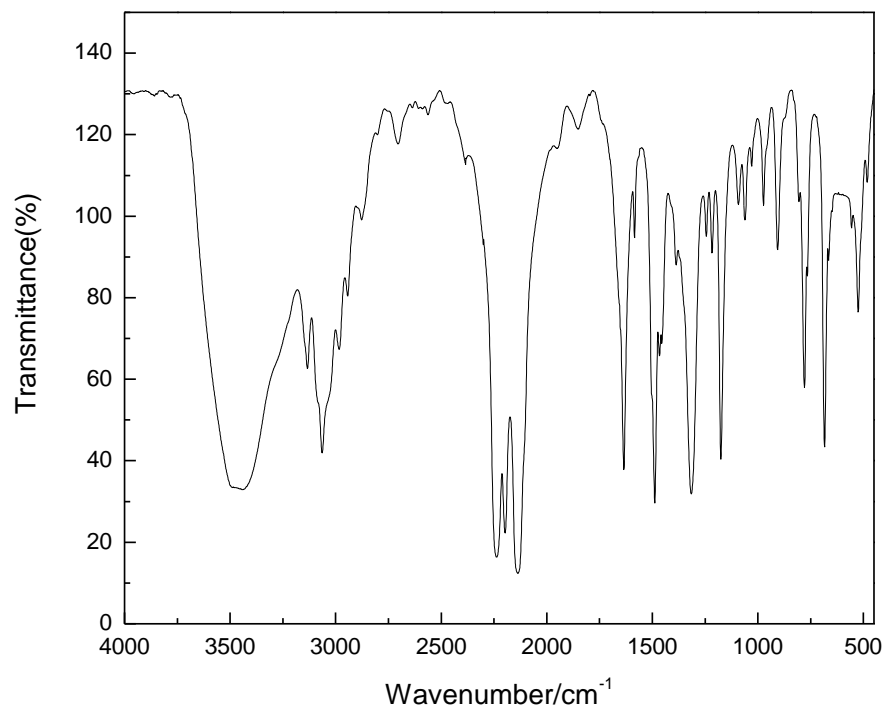

图 S3 离子液体 $\left[\mathrm{C}_{6} \mathrm{py}\right][\mathrm{DCA}]$ 的 FT-IR 谱图

Fig.S3 FT-IR of ILs [C 6 py][DCA]

\section{D 差热扫描量热 DSC 表征}

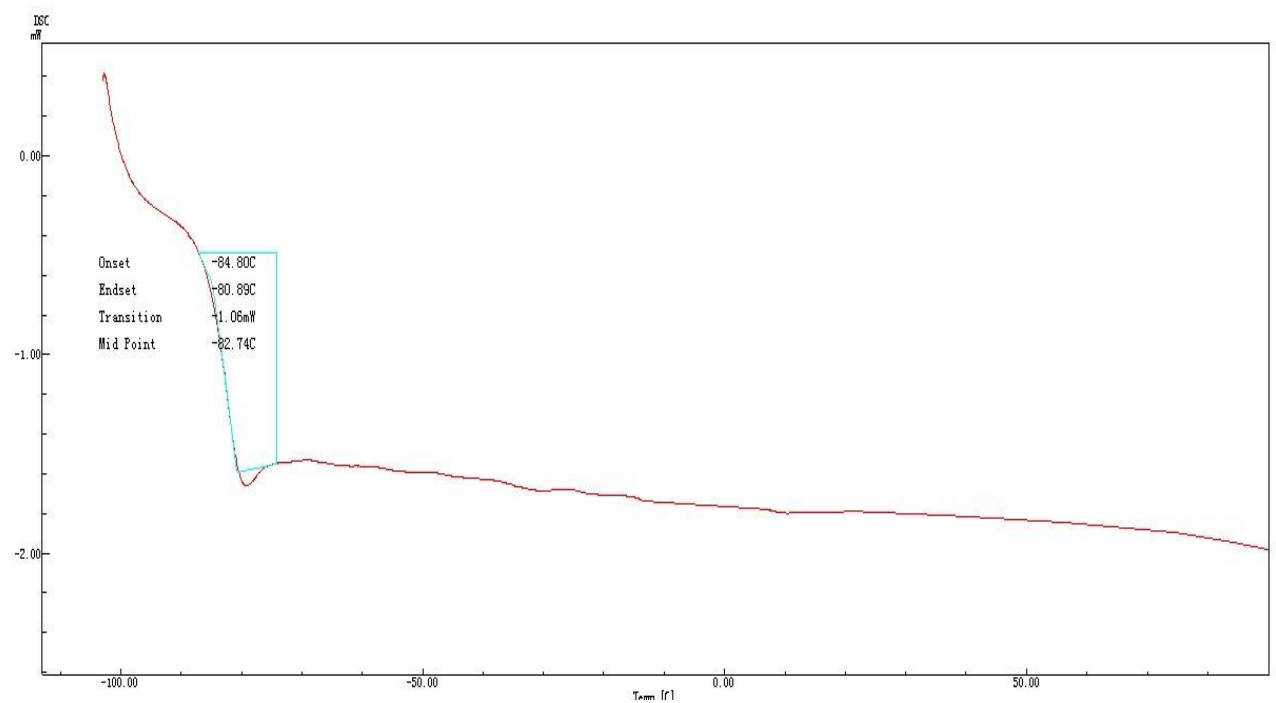

图 S4 离子液体 $\left[\mathrm{C}_{6} \mathrm{py}\right][\mathrm{DCA}]$ 的 DSC 谱图

Fig.S4 DSC trace of IL [ $\left.\mathrm{C}_{6} \mathrm{py}\right][\mathrm{DCA}]$ 
E 高效液相色谱（HPLC）表征

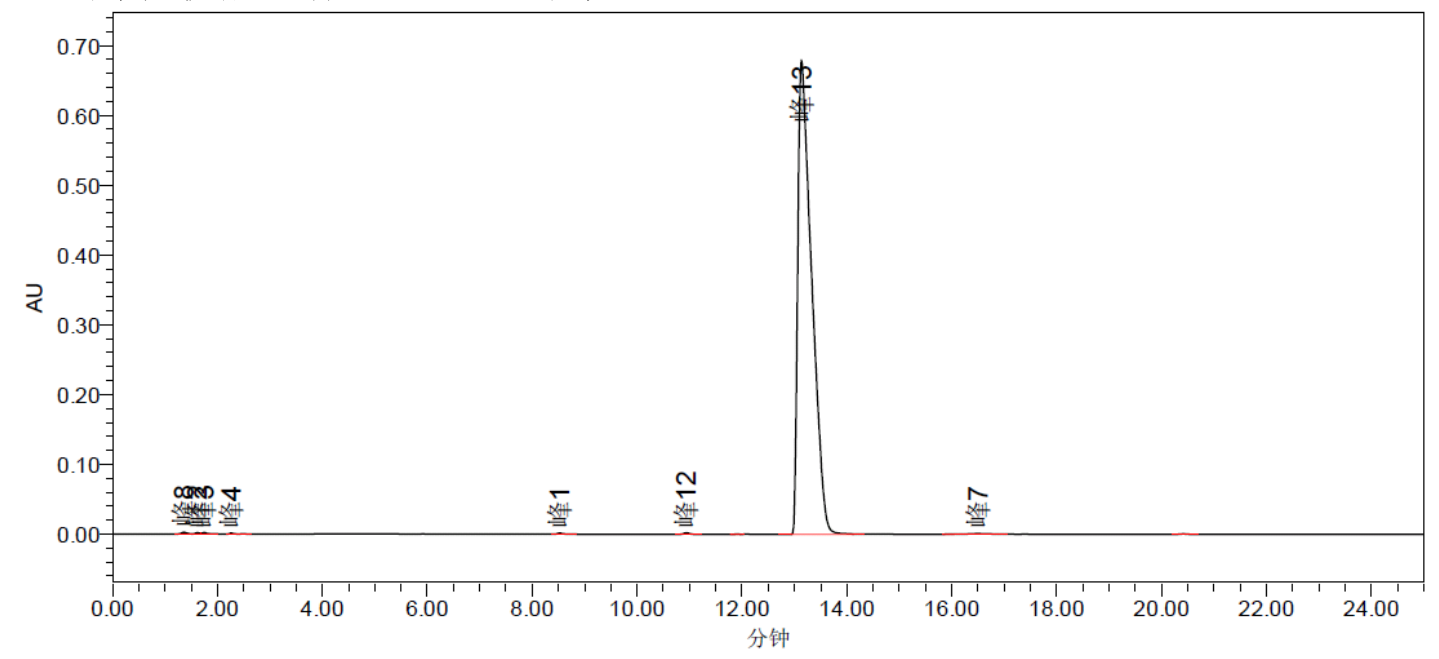

处理通道说明: W2489 ChA 258nm

\begin{tabular}{|c|c|c|c|c|c|c|c|c|c|}
\hline & 名称 & 处理通道说明 & $\begin{array}{c}\text { 保留时间 } \\
\text { (分钟) }\end{array}$ & $\begin{array}{l}\text { 高度 } \\
\text { (微伏) }\end{array}$ & $\begin{array}{c}\text { 面积 } \\
\text { (微伏^秒) }\end{array}$ & 对称因子 & 分离度 & USP 理论塔板数 & $\%$ 面积 \\
\hline 1 & 峰8 & W2489 ChA 258nm & 1.362 & 2595 & 19522 & & & $7.518218 \mathrm{e}+002$ & 0.16 \\
\hline 2 & 峰2 & W2489 ChA 258nm & 1.620 & 1917 & 11069 & & $1.395918 \mathrm{e}+000$ & $1.432314 \mathrm{e}+003$ & 0.09 \\
\hline 3 & 峰3 & W2489 ChA 258nm & 1.750 & 1967 & 13635 & & $7.358148 \mathrm{e}-001$ & $1.444837 \mathrm{e}+003$ & 0.11 \\
\hline 4 & 峰4 & W2489 ChA 258nm & 2.267 & 1112 & 6113 & & $3.258071 \mathrm{e}+000$ & $3.920328 \mathrm{e}+003$ & 0.05 \\
\hline 5 & & W2489 ChA 258nm & 2.491 & 143 & 1090 & & & $1.424404 \mathrm{e}+003$ & 0.01 \\
\hline 6 & 峰5 & W2489 ChA258nm & 4.933 & & & & & & \\
\hline 7 & 峰9 & W2489 ChA258nm & 5.881 & & & & & & \\
\hline 8 & 峰6 & W2489 ChA 258nm & 6.450 & & & & & & \\
\hline 9 & 峰10 & W2489 ChA 258nm & 6.889 & & & & & & \\
\hline 10 & 峰1 & W2489 ChA 258nm & 8.527 & 1546 & 14106 & $1.637852 e+000$ & & $2.234604 \mathrm{e}+004$ & 0.11 \\
\hline 11 & 峰11 & W2489 ChA 258nm & 9.468 & & & & & & \\
\hline
\end{tabular}

处理通道说明: W2489 ChA 258nm

\begin{tabular}{|r|r|r|r|r|r|r|r|r|r|}
\hline & 名称 & 处理通道说明 & $\begin{array}{c}\text { 保留时间 } \\
\text { (分钟) }\end{array}$ & $\begin{array}{c}\text { 高度 } \\
\text { (微伏) }\end{array}$ & $\begin{array}{c}\text { 面积 } \\
\text { (微伏*秒) }\end{array}$ & 对称因子 & 分离度 & USP 理论塔板数 & $\%$ 面积 \\
\hline 12 & 峰12 & W2489 ChA258nm & 10.940 & 2256 & 18802 & $1.169756 \mathrm{e}+000$ & $1.100745 \mathrm{e}+001$ & $4.074485 \mathrm{e}+004$ & 0.15 \\
\hline 13 & & W2489 ChA258nm & 11.908 & 192 & 1185 & $7.158440 \mathrm{e}-001$ & $5.248215 \mathrm{e}+000$ & $7.936148 \mathrm{e}+004$ & 0.01 \\
\hline 14 & & W2489 ChA258nm & 12.839 & 171 & 1147 & & $5.433111 \mathrm{e}+000$ & $7.612358 \mathrm{e}+004$ & 0.01 \\
\hline 15 & 峰13 & W2489 ChA258nm & 13.139 & 680896 & 12429343 & $2.088395 \mathrm{e}+000$ & $8.819239 \mathrm{e}-001$ & $9.611417 \mathrm{e}+003$ & 99.02 \\
\hline 16 & 峰14 & W2489 ChA258nm & 14.097 & & & & & & \\
\hline 17 & & W2489 ChA258nm & 16.083 & 192 & 1592 & & & & 0.01 \\
\hline 18 & 峰7 & W2489 ChA258nm & 16.498 & 816 & 27338 & & & $4.313509 \mathrm{e}+003$ & 0.22 \\
\hline 19 & & W2489 ChA258nm & 20.412 & 834 & 7852 & $1.174663 \mathrm{e}+000$ & $6.525722 \mathrm{e}+000$ & $1.083133 \mathrm{e}+005$ & 0.06 \\
\hline
\end{tabular}

图 S5 离子液体 $\left[\mathrm{C}_{6} \mathrm{py}\right][\mathrm{DCA}]$ 的 HPLC 谱图

Fig.S5 HPLC of IL [ $\mathrm{C}_{6}$ py][DCA] 


\section{二、SAM 作图}

表 S3 288.15 K - 293.15 K 和 $303.15 K-338.15 K^{a}$, [C 6 py][DCA] 不同水含量的密度值 $\rho\left(\mathrm{g} \cdot \mathrm{cm}^{-3}\right)$

Table S5 At $288.15 \mathrm{~K}-293.15 \mathrm{~K}$ and $303.15 \mathrm{~K}-338.15 \mathrm{~K}$, values of density, $\rho\left(\mathrm{g} \cdot \mathrm{cm}^{-3}\right)$, for $\left[\mathrm{C}_{6} \mathrm{py}\right][\mathrm{DCA}]$ contained various amount of water

\begin{tabular}{ccccccccc}
\hline \multicolumn{7}{c}{$\left[\mathrm{C}_{6}\right.$ py][DCA } \\
\hline$T / \mathrm{K}$ & $10^{3} w_{2}=1.78^{b}$ & 3.28 & 4.79 & 6.28 & 7.77 & 0 & $\mathrm{r}$ & $s \times$ \\
288.15 & 1.04226 & 1.04216 & 1.04206 & 1.04195 & 1.04189 & 1.04237 & 0.996 & 1.47 \\
293.15 & 1.03927 & 1.03918 & 1.03908 & 1.03897 & 1.03890 & 1.03938 & 0.998 & 1.06 \\
303.15 & 1.03329 & 1.03321 & 1.03311 & 1.03299 & 1.03291 & 1.03341 & 0.998 & 1.22 \\
308.15 & 1.03030 & 1.03022 & 1.03013 & 1.02999 & 1.02992 & 1.03043 & 0.995 & 1.90 \\
313.15 & 1.02733 & 1.02725 & 1.02715 & 1.02702 & 1.02694 & 1.02746 & 0.997 & 1.51 \\
318.15 & 1.02438 & 1.02430 & 1.02419 & 1.02405 & 1.02397 & 1.02452 & 0.996 & 1.83 \\
323.15 & 1.02144 & 1.02136 & 1.02124 & 1.02111 & 1.02102 & 1.02158 & 0.997 & 1.54 \\
328.15 & 1.01851 & 1.01842 & 1.01831 & 1.01817 & 1.01808 & 1.01865 & 0.997 & 1.51 \\
333.15 & 1.01560 & 1.01551 & 1.01539 & 1.01524 & 1.01515 & 1.01575 & 0.997 & 1.82 \\
338.15 & 1.01270 & 1.01261 & 1.01249 & 1.01233 & 1.01224 & 1.01286 & 0.995 & 2.11 \\
\hline
\end{tabular}

标准不确定度 (0.68 置信水平): ${ }^{a} u(T)= \pm 0.01 \mathrm{~K},{ }^{b} u\left(10^{3} w_{2}\right)= \pm 0.02$.

表 S4 288.15 K - 293.15 K 和 $303.15 \mathrm{~K}-338.15 \mathrm{~K}^{a}$, [C $\left.\mathrm{C}_{6} \mathrm{py}\right][\mathrm{DCA}]$ 不同水含量的表面张力值 $\gamma\left(\mathbf{m} \mathbf{J} \cdot \mathbf{m}^{-2}\right)$

Table S4 At 288.15 K - 293.15 K and 303.15 K - 338.15 K, values of surface tension, $\gamma\left(\mathrm{mJ} \cdot \mathrm{m}^{-2}\right)$, for $\left[\mathrm{C}_{6} \mathrm{py}\right][\mathrm{DCA}]$ contained various amount of water

\begin{tabular}{ccccccccc}
\hline \multicolumn{7}{c}{$\left[\mathrm{C}_{6}\right.$ py $][\mathrm{DCA}]$} \\
\hline$T / \mathrm{K}$ & $10^{3} w_{2}=1.90^{b}$ & 3.40 & 4.89 & 6.39 & 7.91 & 0 & $\mathrm{r}$ & $s \times 10^{5}$ \\
288.15 & 50.3 & 50.5 & 50.7 & 51.0 & 51.3 & 49.9 & 0.995 & 4.71 \\
293.15 & 50.0 & 50.2 & 50.4 & 50.7 & 51.0 & 49.6 & 0.995 & 4.71 \\
303.15 & 49.2 & 49.5 & 49.7 & 50.0 & 50.3 & 48.9 & 0.998 & 3.03 \\
308.15 & 48.8 & 49.1 & 49.4 & 49.6 & 49.9 & 48.5 & 0.998 & 3.19 \\
313.15 & 48.5 & 48.8 & 49.0 & 49.3 & 49.6 & 48.2 & 0.998 & 3.03 \\
318.15 & 48.2 & 48.5 & 48.7 & 49.0 & 49.2 & 47.9 & 0.998 & 3.22 \\
323.15 & 48.0 & 48.2 & 48.4 & 48.7 & 48.9 & 47.7 & 0.997 & 3.15 \\
328.15 & 47.7 & 47.9 & 48.1 & 48.4 & 48.6 & 47.4 & 0.997 & 3.15 \\
333.15 & 47.4 & 47.6 & 47.8 & 48.0 & 48.3 & 47.1 & 0.996 & 3.52 \\
338.15 & 47.1 & 47.3 & 47.5 & 47.7 & 48.0 & 46.8 & 0.996 & 3.52 \\
\hline
\end{tabular}

标准不确定度 (0.68 置信水平): ${ }^{a} u(T)= \pm 0.01 \mathrm{~K},{ }^{b} u\left(10^{3} w_{2}\right)= \pm 0.02$ 。

表 S5 288.15 K - 293.15 K 和 $303.15 \mathrm{~K}-338.15 \mathrm{~K}^{a}$, [C 6 py] [DCA]不同水含量的折光率值 $\boldsymbol{n}_{\mathrm{D}}$ Table S5 At 288.15 K - 293.15 K and 303.15 K - 338.15 K, values of refractive index, $n_{\mathrm{D}}$, for $\left[\mathrm{C}_{6} \mathrm{py}\right][\mathrm{DCA}]$ contained various amount of water 


\begin{tabular}{ccccccccc}
\hline \multicolumn{8}{c}{$\left[\mathrm{C}_{6} \mathrm{py}\right][\mathrm{DCA}]$} \\
\hline$T / \mathrm{K}$ & $10^{3} w_{2}=1.90$ & 3.43 & 4.91 & 6.57 & 8.07 & 0 & $\mathrm{r}$ & $s \times 10^{5}$ \\
288.15 & 1.5295 & 1.5292 & 1.5290 & 1.5288 & 1.5285 & 1.5298 & 0.996 & 4.02 \\
293.15 & 1.5279 & 1.5277 & 1.5275 & 1.5273 & 1.5270 & 1.5282 & 0.996 & 3.74 \\
303.15 & 1.5249 & 1.5247 & 1.5243 & 1.5241 & 1.5238 & 1.5253 & 0.994 & 5.74 \\
308.15 & 1.5235 & 1.5233 & 1.5231 & 1.5228 & 1.5226 & 1.5238 & 0.998 & 2.48 \\
313.15 & 1.5219 & 1.5216 & 1.5214 & 1.5212 & 1.5210 & 1.5221 & 0.995 & 3.96 \\
318.15 & 1.5202 & 1.5199 & 1.5197 & 1.5194 & 1.5191 & 1.5205 & 0.998 & 2.85 \\
323.15 & 1.5188 & 1.5186 & 1.5183 & 1.5181 & 1.5179 & 1.5191 & 0.996 & 3.56 \\
328.15 & 1.5174 & 1.5172 & 1.5170 & 1.5168 & 1.5166 & 1.5176 & 0.999 & 0.62 \\
333.15 & 1.5157 & 1.5155 & 1.5152 & 1.5150 & 1.5148 & 1.5160 & 0.996 & 3.56 \\
338.15 & 1.5141 & 1.5139 & 1.5137 & 1.5134 & 1.5132 & 1.5144 & 0.998 & 2.48 \\
\hline 标准不确定度 $(0.68$ 置信水平 $):{ }^{a} u(T)= \pm 0.01 \mathrm{~K},{ }^{b} u\left(10^{3} w_{2}\right)= \pm 0.02 。$ & &
\end{tabular}

标准不确定度 (0.68 置信水平): ${ }^{a} u(T)= \pm 0.01 \mathrm{~K},{ }^{b} u\left(10^{3} w_{2}\right)= \pm 0.02$ 。

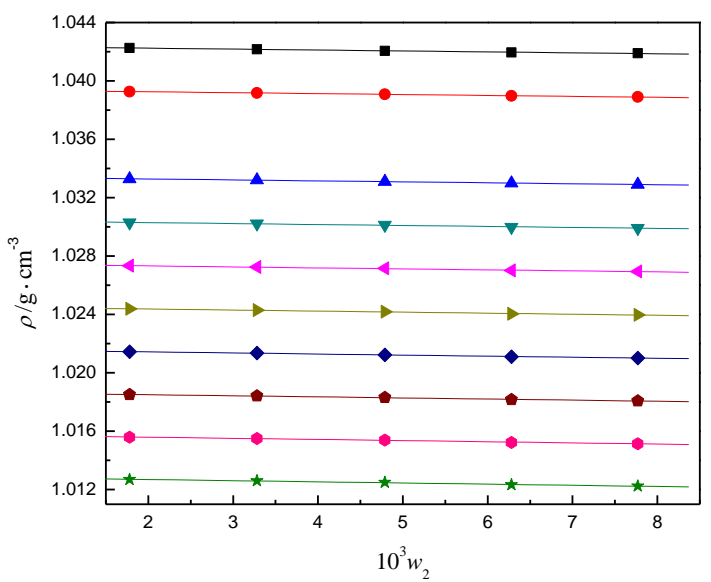

图 S6 [C 6 py] [DCA]的密度值对其含水量作图

Fig.S6 The plot of density vs. the various amount of water in [ $\left.\mathrm{C}_{6} \mathrm{py}\right][\mathrm{DCA}]$

D28.15 K: $\rho=1.04237-0.63424 \times 10^{-4} w_{2}, s=1.47 \times 10^{-5}, r=0.996$;

$293.15 \mathrm{~K}: \rho=1.03938-0.63422 \times 10^{-4} w_{2}, s=1.06 \times 10^{-5}, r=0.998$;

$\triangle 303.15 \mathrm{~K}: \rho=1.03341-0.65418 \times 10^{-4} w_{2}, s=1.22 \times 10^{-5}, r=0.998$;

$\nabla 308.15 \mathrm{~K}: \rho=1.03043-0.66082 \times 10^{-4} w_{2}, s=1.90 \times 10^{-5}, r=0.995$;

$4313.15 \mathrm{~K}: \rho=1.02746-0.67419 \times 10^{-4} w_{2}, s=1.51 \times 10^{-5}, r=0.997$;

$318.15 \mathrm{~K}: \rho=1.02452-0.71425 \times 10^{-4} w_{2}, s=1.83 \times 10^{-5}, r=0.996$;

$323.15 \mathrm{~K}: \rho=1.02158-0.72762 \times 10^{-4} w_{2}, s=1.54 \times 10^{-5}, r=0.997$

$328.15 \mathrm{~K}: \rho=1.01865-0.74095 \times 10^{-4} w_{2}, s=1.51 \times 10^{-5}, r=0.997$;

$333.15 \mathrm{~K}: \rho=1.01575-0.78101 \times 10^{-4} w_{2}, s=1.83 \times 10^{-5}, r=0.997$

$\star 338.15 \mathrm{~K}: \rho=1.01286-0.80102 \times 10^{-4} w_{2}, s=2.11 \times 10^{-5}, r=0.995$. 


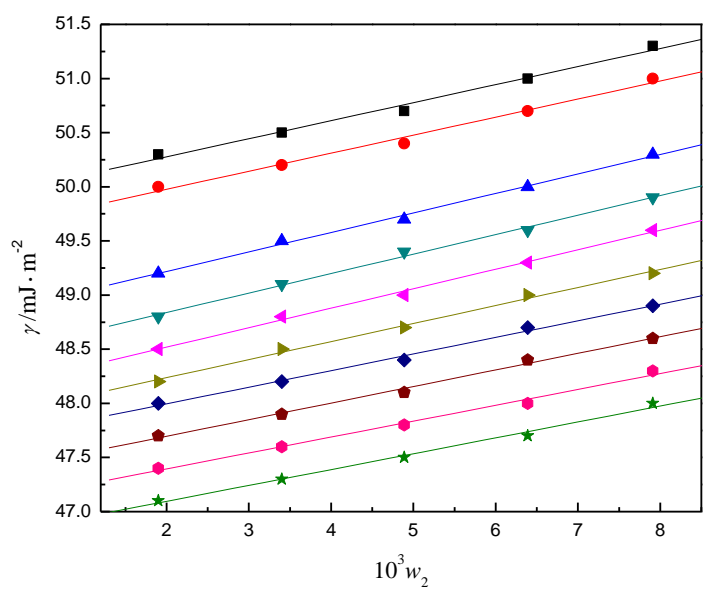

图 S7 [C 6 py][DCA]的表面张力对其含水量作图

Fig.S7 The plot of surface tension vs. the various amount of water in [ $\left.\mathbf{C}_{6} \mathrm{py}\right][\mathrm{DCA}]$

$288.15 \mathrm{~K}: \gamma=49.96+0.1666 w_{2}, s=3.11 \times 10^{-2}, r=0.998$;

$0293.15 \mathrm{~K}: \gamma=49.72+0.1599 w_{2}, s=3.57 \times 10^{-2}, r=0.997$;

$\triangle 303.15 \mathrm{~K}: \gamma=48.86+0.1799 w_{2}, s=3.03 \times 10^{-2}, r=0.998$;

$\nabla 308.15 \mathrm{~K}: \gamma=48.48+0.1799 w_{2}, s=3.19 \times 10^{-2}, r=0.998$;

$\angle 313.15 \mathrm{~K}: \gamma=48.16+0.1799 w_{2}, s=3.03 \times 10^{-2}, r=0.998$;

$318.15 \mathrm{~K}: \gamma=47.90+0.1665 w_{2}, s=3.22 \times 10^{-2}, r=0.998$;

$\checkmark 323.15 \mathrm{~K}: \gamma=47.69+0.1532 w_{2}, s=3.15 \times 10^{-2}, r=0.997$;

$328.15 \mathrm{~K}: \gamma=47.39+0.1532 w_{2}, s=3.15 \times 10^{-2}, r=0.997$;

$333.15 \mathrm{~K}: \gamma=47.10+0.1466 w_{2}, s=3.52 \times 10^{-2}, r=0.996$;

$\star 338.15 \mathrm{~K}: \gamma=46.80+0.1466 w_{2}, s=3.52 \times 10^{-2}, r=0.996$.

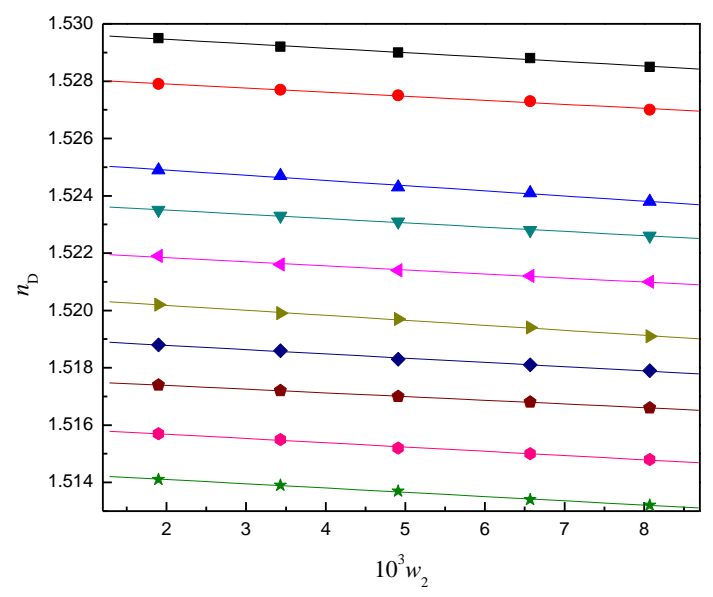

图 S8 [C 6 py] [DCA]的折光率对其含水量作图

Fig.S8 The plot of refractive index $v s$. the various amount of water in [ $\left.\mathrm{C}_{6} \mathrm{py}\right]$ [DCA]

$288.15 \mathrm{~K}: n_{\mathrm{D}}=1.5298-1.5490 \times 10^{-4} w_{2}, s=4.02 \times 10^{-5}, r=0.996$;

$293.15 \mathrm{~K}: n_{\mathrm{D}}=1.5282-1.4207 \times 10^{-4} w_{2}, s=3.74 \times 10^{-5}, r=0.996$;

$\Delta 303.15 \mathrm{~K}: n_{\mathrm{D}}=1.5253-1.8064 \times 10^{-4} w_{2}, s=5.74 \times 10^{-5}, r=0.994$; 
$\nabla 308.15 \mathrm{~K}: n_{\mathrm{D}}=1.5238-1.4872 \times 10^{-4} w_{2}, s=2.48 \times 10^{-5}, r=0.998$;

$4313.15 \mathrm{~K}: n_{\mathrm{D}}=1.5221-1.4200 \times 10^{-4} w_{2}, s=3.96 \times 10^{-5}, r=0.995$;

$318.15 \mathrm{~K}: n_{\mathrm{D}}=1.5205-1.7446 \times 10^{-4} w_{2}, s=2.85 \times 10^{-5}, r=0.998$;

$\checkmark 323.15 \mathrm{~K}: n_{\mathrm{D}}=1.5191-1.4844 \times 10^{-4} w_{2}, s=3.56 \times 10^{-5}, r=0.996$;

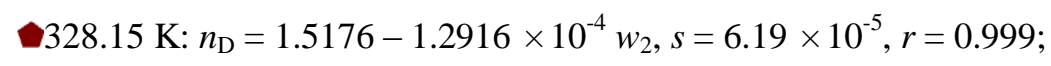

$333.15 \mathrm{~K}: n_{\mathrm{D}}=1.5160-1.4844 \times 10^{-4} w_{2}, s=3.56 \times 10^{-5}, r=0.996$;

$\star 338.15 \mathrm{~K}: n_{\mathrm{D}}=1.5144-1.4872 \times 10^{-4} w_{2}, s=2.48 \times 10^{-5}, r=0.998$. 